\title{
A CLASS OF COMMUTATIVE BANACH ALGEBRAS WITH UNIQUE COMPLETE NORM TOPOLOGY AND CONTINUOUS DERIVATIONS
}

\author{
JOHN A. LINDBERG, JR.
}

AbSTRACT. Let $A$ be a semisimple commutative complex algebra with identity and $\alpha(x)$ a monic polynomial over $A$. Two results are proved. If $B=A[x] /(\alpha(x))$ is a Banach algebra under some norm, then $B$ has a unique complete norm topology. Furthermore, $B$ has nontrivial derivations if and only if $B$ has a nontrivial radical.

Introduction. It is known that if $A$ is a commutative Banach algebra with identity and $\alpha(x)$ is a monic polynomial over $A$, then $B=A[x] /(\alpha(x))$ can be made into a Banach algebra with a norm extending the norm on $A[1]$. In [2], D. T. Brown has shown that if $A$ is semisimple and regular (in the sense of Silov), then $B$ has unique complete norm topology. The assumption of regularity can be dropped as we show in $\$ 1$. In general, the extension $B$ is not semisimple; indeed, $B$ has a nontrivial radical if and only if the discriminant of $\alpha(x)$ is zero or a zero divisor in $A$ [5].

$\$ 2$ is motivated by a recent result of $\mathrm{R}$. J. Loy that states, in our context, that all derivations on $B$ are continuous [4]. However, he does not show the existence of derivations on (nonsemisimple) $B$ even though he gives their form. We consider the existence of nontrivial derivations in $\$ 2$.

1. Uniqueness of complete norm topology on $A[x] /(\alpha(x))$. It is convenient to set first some of our notation and to make several comments. Lemma 1 of [2] states that if $A$ is a semisimple algebra (all algebras in this paper are assumed to be over the complex field $C$ and to possess an identity element) and if $B=A[x] /(\alpha(x))$ is a Banach algebra, then $A$ is a Banach algebra under some norm. Actually, it is proved that if $K(A)$ denotes the intersection of the kernels of nontrivial complex homomorphisms, then $A / K(A)$ is a Banach algebra. However, it is known that each maximal ideal $M$ in $A$ is contained in a maximal ideal $M_{0}$ in $B$ such that $M=M_{0} \cap A$ (see p. 259 of [7]). Since $B$ is a Banach algebra, $M_{0}$ is the kernel of a com-

Received by the editors June 26, 1970.

AMS 1969 subject classifications. Primary 4655, 4650; Secondary 1360.

Key words and phrases. Uniqueness of complete norm topology, continuous derivations, normed extensions, commutative Banach algebras.

Copyright (c) 1971, American Mathematical Society 
plex homomorphism; hence, so is $M$. Thus, $K(A)=(0)$ since $A$ is semisimple and Brown's Lemma 1 follows.

For a complex algebra $A$, let $\Phi_{A}$ denote the space of nontrivial complex homomorphisms on $A$, together with the Gelfand topology (see Chapter III of [6]). If $p(x) \in A[x]$, let $p(x)$ denote the coset $p(x)+(\alpha(x))$, and if $\phi \in \Phi_{A}$, let $p_{\phi}(x)$ denote $\sum \phi\left(p_{i}\right) x^{i}, p(x)=\sum p_{i} x^{i}$. The space $\Phi_{B}$ is identifiable with $\left\{(\phi, \lambda) \in \Phi_{A} \times C: \alpha_{\phi}(\lambda)=0\right\}$. The action of $(\phi, \lambda) \in \Phi_{B}$ on $p(\mathbf{x})$ is given by $(\phi, \lambda)(p(\mathbf{x}))=p_{\phi}(\lambda)$.

Finally, before proceeding to the theorem of this section, we discuss a recent result of $\mathrm{B}$. E. Johnson which will be used in our proof. In the setting of a Banach algebra, he proved in [3] that if $d$ is a derivation of the Banach algebra $A$ into the bounded functions on $\Phi_{A}$, then the point derivations $d_{\phi}$ defined by $\phi \circ d$ are continuous on $A$ for all but at most a finite number of $\phi$ 's. Now, suppose that at each point $\phi \in \Phi_{A}$ we have a finite number of point derivations, but no more than $n$, some positive number. Let them be denoted by $d_{\phi}^{1}, \cdots, d_{\phi}^{k_{\phi}}, k_{\phi} \leqq n, \phi \in \Phi_{A}$. If $d_{\phi}^{i}(a)$ is bounded for all $i, \phi$ and $a \in A$, then by at most $n$ applications of Johnson's result, we have that for all $i$ and for all except possibly a finite number of $\phi$ 's, $d_{\phi}^{i}$ is continuous on $A$.

Theorem 1. Let $A$ be a semisimple complex algebra and $\alpha(x)$ a monic polynomial of degree $n$ over $A$. If $B=A[x] /(\alpha(x))$ is a Banach algebra with norm $\|\cdot\|$, then $A$ is $\|\cdot\|$-closed in $B$. Consequently, $B$ has unique complete norm topology.

Proof. By the comments preceding the statement of the theorem, we can conclude that each $\phi \in \Phi_{A}$ is $\|\cdot\|$-continuous on $A$, where $\|\cdot\|$ is the norm on $B$. Thus, $\Phi_{\bar{A}}=\Phi_{A}, \bar{A}$ the $\|\cdot\|$-closure of $A$ in $B$.

Suppose $\left\{a_{n}\right\}$ is a sequence in $A$ converging to $p(x) \in B$ with respect to $\|\cdot\|$. Then $\phi\left(a_{n}\right)=(\phi, \lambda)\left(a_{n}\right) \rightarrow(\phi, \lambda)(p(x))=\phi(p(\lambda))$ for all $(\phi, \lambda) \in \Phi_{B}$. This implies that there exists an $a \in A$ such that $\phi\left(a_{n}\right)$ $\rightarrow \phi(a)$ for all $\phi \in \Phi_{A}$. The existence of such an $a$ is shown in the proof of Theorem 3 in [2]. Hence, $p(x)$ can be uniquely expressed as the sum of an element in $A$ and an element in the radical $R(B)$ of $B$, namely, $p(\mathbf{x})=a+[p(\mathbf{x})-a]$ and $p(\mathbf{x})-a \in \bar{A} \cap R(B)$.

We next show that there is a finite subset $F$ of $\Phi_{B}$ such that if $p(\mathbf{x})$ $\in \bar{A}, \partial p \leqq n-1$, then $\phi\left(p^{(j-1)}(\lambda)\right)=0$ for $j$ satisfying $2 \leqq j \leqq M(\phi, \lambda)$ and for all $(\phi, \lambda) \in \Phi_{B} \backslash F$, where $M(\phi, \lambda)$ denotes the multiplicity of $\lambda$ as a root of $\alpha_{\phi}(x)=0$. For $j \geqq 1, p^{(j)}(x)$ denotes the $j$ th derivative of $p(x)$ and $p^{(0)}(x)=p(x)$. Consider the statement: there exists a finite subset $F_{i} \subset \Phi_{B}$ such that $2 \leqq j \leqq \min (i, M(\phi, \lambda))$ implies $\phi\left(p^{(j-1)}(\lambda)\right)=0$ for all $p(x) \in \bar{A}$ with $\partial p \leqq n-1$ and for all $(\phi, \lambda) \in \Phi_{B} \backslash F_{i}$. Suppose that 
the statement is true for $i$. We proceed to show that it is true for $i+1$ as well. Define $d_{(\phi, \lambda)}$ for $(\phi, \lambda) \in \Phi_{B}$ as follows:

$$
\begin{aligned}
d_{(\phi, \lambda)}(p(\mathbf{x})) & =0 & & \text { if } M(\phi, \lambda) \leqq i \text { or }(\phi, \lambda) \in F_{i}, \\
& =\phi\left(p^{(i)}(\lambda)\right) & & \text { otherwise, }
\end{aligned}
$$

where $p(x) \in \bar{A}$ and $\partial p \leqq n-1$. Clearly, for each $p(\mathbf{x}) \in \bar{A}$ with $\partial p \leqq n-1$,

$$
\sup _{(\phi, \lambda) \in \Phi_{B}}\left|d_{(\phi, \lambda)}(p(\mathbf{x}))\right|<+\infty,
$$

and $d_{(\phi, \lambda)}$ is a linear functional on $\bar{A}$. To show that $d_{(\phi, \lambda)}$ is a point derivation at $\phi$ on $\bar{A}$, let $p(\mathbf{x}), q(\mathbf{x}) \in \bar{A}, \partial p \leqq n-1, \partial q \leqq n-1$ and write $p(x) q(x)=Q(x) \alpha(x)+r(x)$, where $Q(x), r(x) \in A[x]$ and $\partial r \leqq n-1$. If $M(\phi, \lambda) \leqq i$ or $(\phi, \lambda) \in F_{i}, d_{(\phi, \lambda)}=0$ and there is nothing to prove. Suppose, therefore, $M(\phi, \lambda) \geqq i+1$ and $(\phi, \lambda) \notin F_{i}$. Then $d_{(\phi, \lambda)}(p(\mathbf{x}) q(\mathbf{x}))=\phi\left(r^{(i)}(\lambda)\right)$. But

$$
r^{(i)}(x)=\sum_{j=0}^{i}\left(\begin{array}{l}
i \\
j
\end{array}\right)\left\{p^{(i-j)}(x) q^{(j)}(x)-Q^{(i-j)}(x) \alpha^{(j)}(x)\right\} .
$$

Thus,

$$
\begin{aligned}
d_{(\phi, \lambda)}(p(\mathbf{x}) q(\mathbf{x})) & =\sum_{j=0}^{i}\left(\begin{array}{l}
i \\
j
\end{array}\right)\left\{\phi\left(p^{(i-j)}(\lambda)\right) \phi\left(q^{(j)}(\lambda)\right)-\phi\left(Q^{(i-j)}(\lambda)\right) \phi\left(\alpha^{(i)}(\lambda)\right)\right\} \\
& =\phi\left(p^{(i)}(\lambda)\right) \phi(q(\lambda))+\phi(p(\lambda)) \phi\left(q^{(i)}(\lambda)\right) \\
& =d_{(\phi, \lambda)}(p(\mathbf{x}))(\phi, \lambda)(q(\mathbf{x}))+(\phi, \lambda)(p(\mathbf{x})) d_{(\phi, \lambda)}(q(\mathbf{x}))
\end{aligned}
$$

since $\phi\left(\alpha^{(j)}(\lambda)\right)=0$ for $j=0,1,2, \cdots, i$ and $p(x), q(x) \in \bar{A}, \partial p \leqq n-1$, $\partial q \leqq n-1, \quad(\phi, \lambda) \notin F_{i}$ imply that $\phi\left(p^{(j-1)}(\lambda)\right)=\phi\left(q^{(j-1)}(\lambda)\right)=0$ for $j=2, \cdots, \min (i, M(\phi, \lambda))=i$. Hence, $d_{(\phi, \lambda)}$ is a point derivation. By the extension of Johnson's result mentioned above, we can conclude that there is a finite set $F_{i+1}$ such that $d_{(\phi, \lambda)}$ is continuous whenever $(\phi, \lambda) \in \Phi_{B} \backslash F_{i+1}$. We may and do assume that $F_{i+1} \supset F_{i}$. Now, if

$$
a_{n} \stackrel{\|\cdot\|}{\longrightarrow} p(\mathrm{x}), \quad\left\{a_{n}\right\} \subset A,
$$

then $d_{(\phi, \lambda)}\left(a_{n}\right)=0$ for all $n$ so that $(\phi, \lambda)\left(p^{(i)}(\mathbf{x})\right)=0$ whenever $M(\phi, \lambda)$ $\geqq i+1$ and $(\phi, \lambda) \notin F_{i+1}$. Thus $2 \leqq j \leqq \min (i+1, M(\phi, \lambda))$ implies that $\phi\left(p^{(j-1)}(\lambda)\right)=0$ whenever $(\phi, \lambda) \notin F_{i+1}$. Since the argument also shows that the statement is true for $i=2$, we have by induction that $\phi\left(p^{(j-1)}(\lambda)\right)=0$ whenever $2 \leqq j \leqq M(\phi, \lambda)$, except possibly for $(\phi, \lambda)$ belonging to a finite subset $F$ of $\Phi_{B}$. (For the case $i=2, d_{(\phi, \lambda)}(p(x))$ is simply $\phi\left(p^{(1)}(\lambda)\right)$ when $2 \leqq M(\phi, \lambda)$ and zero otherwise.) 
Let $F^{\prime}=(\pi)^{-1}(\pi(F))$, and let $p(x) \in \bar{A} \cap R(B)$, where $\pi(\phi, \lambda)=\phi$ for $(\phi, \lambda) \in \Phi_{B}$. Then $(\phi, \lambda) \in \Phi_{B}$ implies that $\phi(p(\lambda))=0$. We may assume that the degree $\partial p$ of $p(x)$ is smaller than $n$. Now, suppose that $(\phi, \lambda) \notin F^{\prime}$ and that $p_{\phi}(x)$ is not the zero polynomial. Then $\lambda$ is a root of $p_{\phi}(x)=0$ of at least multiplicity $M(\phi, \lambda)$. But this implies that $p_{\phi}(x)=0$ has at least $n=\sum_{(\phi, \lambda) \in(\pi)^{-1}(\phi)} M(\phi, \lambda)$ roots, each repeated according to multiplicty. This is a contradiction since $\partial p \leqq n-1$. Hence $(\phi, \lambda) \notin F^{\prime}$ implies that $p_{\phi}(x)$ is the zero polynomial. Thus, if $p(x)=\sum_{k=0}^{n-1} p_{k} x^{k} \in R(B) \cap \bar{A}$, then each $\hat{p}_{k}$ vanishes on $\Phi_{A}$, except possibly for a finite subset. If $\hat{p}_{k}(\phi) \neq 0$, then $\phi$ is an isolated point of $\Phi_{\Lambda}$.

We now continue as in [2]. If $\phi$ is an isolated point in $\Phi_{A}$, then there exists an idempotent $u_{\phi}$ in $A$ such that $\tilde{u}_{\phi}(\theta)=1$ if and only if $\theta=\phi$ (see $[6$, p. 168]), since $A$ is a Banach algebra under some norm (see [2, Lemma 1]). Since $A$ is semisimple $u_{\phi} a=\phi(a) u_{\phi}, a \in A$, and therefore $u_{\phi} A$ is closed in $B$. Now, if $p(x)=p_{0}+\cdots+p_{n-1} x^{n-1} \in B$, then $u_{\phi} p(\mathbf{x})=\phi\left(p_{0}\right) u_{\phi}+\phi\left(p_{1}\right) u_{\phi} \mathbf{x}+\cdots+\phi\left(p_{n-1}\right) u_{\phi} \mathbf{x}^{n-1}$. Furthermore if

$$
a_{n} \stackrel{\|\cdot\|}{\longrightarrow} p(\mathbf{x})
$$

then $u_{\phi} a_{n} \rightarrow u_{\phi} p(x)$ and $u_{\phi} p(x)$ must lie in $u_{\phi} A$. Hence, $u_{\phi} p_{k}=0$, $1 \leqq k \leqq n-1$, or equivalently, $\phi\left(p_{k}\right)=0$ for $1 \leqq k \leqq n-1$. Thus, $p(x) \in \bar{A}$ implies that $p(x)=p_{0} \in A$ and $A=\bar{A}$ since $A$ is semisimple.

The second assertion follows from Theorem 2 of [2].

2. Derivations on $A[x] /(\alpha(x))$. In our context, the work of Loy in [4] shows that if $A$ is a semisimple Banach algebra, then any derivation $D$ on $B=A[x] /(\alpha(x))$ must be of the form $D(p(x))=p^{\prime}(\mathbf{x}) D(x)$, $p(x)=p_{0}+p_{1} x+\cdots+p_{n-1} x^{n-1}, n=\partial \alpha$. This particularly simple form reflects the fact that $A$ has no nontrivial derivations into itself [3]. It is easily established that any linear transformation on $B$ of the form $p(\mathbf{x}) \rightarrow p^{\prime}(\mathbf{x}) p_{0}(\mathbf{x}), p_{0}(\mathbf{x})$ fixed, is a derivation on $B$ if and only if $\alpha^{\prime}(\mathbf{x}) p_{0}(\mathbf{x})=0$. Note that $p(x) \rightarrow p^{\prime}(x) p_{0}(x)$ is continuous with respect to any Banach algebra norm on $B$, by virtue of Theorem 1 .

THEOREM 2. Let $A$ be a semisimple commutative Banach algebra and $\alpha(x) \in A[x]$ a monic polynomial. Then $B$ has a nontrivial derivation if and only if the radical of $B$ is nontrivial.

Proof. The only if part follows from Theorem 4 of [3].

Suppose, now, that $R(B) \neq(0)$. By Corollary 9.3 of [5], we have that the discriminant $d_{\alpha}$ of $\alpha(x)$ is either zero or a zero divisor in $A$, say $d_{\alpha} c=0, c \neq 0$. From Lemma 9.1 of [5], we have the existence of 
polynomials $\gamma(x), \delta(x), R(x) \in A[x]$ and nonzero elements $a, b \in A$ such that

(i) $a \alpha(x)=\gamma(x) R(x)$,

(ii) $b \alpha^{\prime}(x)=\delta(x) R(x)$,

(iii) $\phi(a)=0$ if and only if $\phi(b)=0$, and $\phi(c)=0$ implies $\phi(a)=0$, and

(iv) if $\beta_{\phi}(x)\left|\alpha_{\phi}(x), \beta_{\phi}(x)\right| \alpha_{\phi}^{\prime}(x)$, then $\beta_{\phi}(x) \mid R_{\phi}(x)$, where $\phi \in \Phi_{A}$.

Set $p_{0}(\mathbf{x})=a b \gamma(\mathbf{x})$, and $D(p(\mathbf{x}))=p^{\prime}(\mathbf{x}) p_{0}(\mathbf{x})$. To show that $D$ is a derivation, we need only verify that $\alpha^{\prime}(x) p_{0}(x)=0$, that is, $\alpha^{\prime}(x) p_{0}(x)$ $\in(\alpha(x))$. By conditions (i) and (ii), we have that

$$
p_{0}(x) \alpha^{\prime}(x)=a b \gamma(x) \alpha^{\prime}(x)=a \gamma(x) \delta(x) R(x)=a^{2} \delta(x) \alpha(x) .
$$

We next show that $D$ is nontrivial.

Suppose that $p_{0}(x)=q(x) \alpha(x)$. Then $q(x) \alpha^{\prime}(x) \alpha(x)=a^{2} \delta(x) \alpha(x)$. Since $\alpha(x)$ is monic and hence not a zero divisor in $A[x], q(x) \alpha^{\prime}(x)$ $=a^{2} \delta(x)$. Now, $a^{2} b \alpha^{\prime}(x)=a^{2} \delta(x) R(x)=q(x) \alpha^{\prime}(x) R(x)$. Hence, $a^{2} b$ $=q(x) R(x)$. If $\phi \in \Phi_{A}$ and $\phi(a) \neq 0$, then $q_{\phi}(x) R_{\phi}(x)=\phi\left(a^{2} b\right) \neq 0$ by (iii), and consequently, $q_{\phi}(x)$ and $R_{\phi}(x)$ are constant polynomials. On the other hand, if $\phi \in \Phi_{A}$ and $\phi(a) \neq 0$, then $\phi(c) \neq 0$. Therefore, $\phi\left(d_{\alpha}\right)=0$ so that there exists a complex number $\lambda$ such that $(x-\lambda) \mid \alpha_{\phi}(x)$ and $(x-\lambda) \mid \alpha_{\phi}^{\prime}(x)$. By (iv), $(x-\lambda) \mid R_{\phi}(x)$ so that $\partial R_{\phi}(x)>0$, a contradiction. It follows that $p_{0}(x) \notin(\alpha(x))$, and $D$ as defined is not trivial. This completes the proof.

In conclusion, we note that since the results from [5] used above are valid for semisimple complex algebras, Theorem 2 is true for semisimple complex algebras $A$ without nontrivial derivations.

\section{REFERENCES}

1. R. Arens and K. Hoffman, Algebraic extension of normed algebras, Proc. Amer. Math. Soc. 7 (1956), 203-210. MR 17, 1113.

2. D. T. Brown, $A$ class of Banach algebras with a unique norm topology, Proc. Proc. Amer. Math. Soc. 17 (1966) 1429-1434. MR 34 \#4938.

3. B. E. Johnson, Continuity of derivations on commutative algebras, Amer. J. Math. 91 (1969), 1-10. MR 39 \#7433.

4. R. J. Loy, Uniqueness of the complete norm topology and continuity of derivations on Banach algebras, Tôhoku Math. J. 22 (1970), 371-378.

5. J. A. Lindberg, Algebraic extensions of commutative Banach algebras, Pacific J. Math. 14 (1964), 559-583. MR $30 \# 3380$.

6. C. E. Rickart, General theory of Banach algebras, University Series in Higher Math., Van Nostrand, Princeton, N. J., 1960. MR 22 \#5903.

7. O. Zariski and P. Samuel, Commutative algebra. Vol. I, University Series in Higher Math., Van Nostrand, Princeton, N. J., 1958. MR 19, 833.

Syracuse University, Syracuse, New York 13210 